\title{
Plastome Diversity and Phylogenomic Relationships in Asteraceae
}

\author{
Joan Pere Pascual-Díaz ${ }^{1}$, Sònia Garcia 1,* and Daniel Vitales 1,2,* \\ 1 Institut Botànic de Barcelona (IBB-CSIC), Passeig del Migdia s/n, 08038, Barcelona, Catalonia, Spain; joan- \\ pere.pascual@ibb.csic.es; soniagarcia@ibb.csic.es; daniel.vitales@ibb.csic.es \\ 2 Laboratori de Botànica - Unitat associada CSIC, Facultat de Farmàcia i Ciències de l'Alimentació, Universi- \\ tat de Barcelona, Av. Joan XXIII 27-31, 08028, Barcelona, Catalonia, Spain; daniel.vitales@ibb.csic.es \\ * Correspondence: daniel.vitales@ibb.csic.es, soniagarcia@ibb.csic.es; Tel.: +34 93289611
}

\begin{abstract}
Plastid genomes are in general highly conserved given their slow evolutionary rate, thus large changes in their structure are unusual. However, when specific rearrangements are present, they are often phylogenetically informative. Asteraceae is a highly diverse family whose evolution is long driven by polyploidy (up to 48x) and hybridisation, both processes usually complicating systematic inferences. In this study, we have generated one of the most comprehensive plastomebased phylogenies of family Asteraceae, providing information about the structure, genetic diversity, and repeat composition of these sequences. By comparing the whole plastome sequences obtained, we confirmed the double inversion located in the long single copy region, for most of the species analysed (with the exception of basal tribes), a well-known feature for Asteraceae plastomes. We also show that genome size, gene order and gene content are highly conserved along the family. However, species representative of the basal subfamily Barnadesioideae -as well as in the sister family Calyceraceae - are lacking the pseudogene rps19 located in one inverted repeat. The phylogenomic analysis conducted here, based on 63 protein-coding genes, 30 transfer RNA genes and 21 ribosomal RNA genes from 36 species of Asteraceae, are overall consistent with the general consensus for the family's phylogeny, while resolving the position of tribe Senecioneae and revealing some incongruences at tribe level between reconstructions based on nuclear and plastid DNA data.
\end{abstract}

Keywords: chloroplast genome, Compositae, phylogenetic incongruence, plastid DNA, Senecioneae

\section{Introduction}

The sunflower family (Asteraceae or Compositae) is probably the most diversified of plants, with about 25,000-35,000 species, being distributed worldwide and accounting for ca. $10 \%$ of angiosperms $[1,2]$. The family contains many important crops (such as lettuce, sunflower or artichoke), many ornamentals (such as marigolds or dahlias), but also many weeds (such as dandelion or some thistles) [1]. Two of the defining morphological traits of the family have been crucial for the evolutionary and ecological success of Asteraceae: the characteristic inflorescence in capitulum, in which many tiny flowers (florets) are packed in a receptacle; and the cypsela, an indehiscent dry fruit derived from a compound inferior ovary, which usually has adaptations for an effective dispersal and to herbivory $[3,4]$. Asteraceae has long been the subject of cytological interest and it is possibly the plant family for which more chromosome counts are available, with $x=9$ as the most likely ancestral base number. Hybridisation and polyploidy are particularly active in the family, with ploidy levels up to 48x [5], and indeed several whole genome duplications (WGD) [6], together with frequent hybridisation phenomena [7,8], are linked to the massive diversification of Asteraceae, complicating, at the same time, its systematics. Other cytological features, such as the presence of an exceptional linked arrangement of ribosomal RNA genes in many of its species [9] add interest to the study of this family. 
As already supported by early molecular phylogenetic studies [10], Asteraceae constitutes a well-defined family that originated in South America, its most closely related families being Calyceraceae (South American origin) and Goodeniaceae (Southwestern Australian origin) $[11,12]$. Resolving the systematic relationships within Asteraceae has been, however, a more challenging task. Since the first molecular-based approaches $[13,14]$ to the major compilation and meta-tree analyses by [1], plastid DNA has been a preferred target of researchers interested in Asteraceae evolution. The combination of slowly evolving genic regions and the fast evolutionary rate of intergenic spacers have made plastid markers classical candidates for phylogenetic reconstruction at different taxonomic levels within the family [15]. One of the latest and most comprehensive evolutionary reconstructions at the family level was based on DNA sequences of 11 plastid genes [16]. More recently, the backbone of Asteraceae phylogeny was successfully resolved using "targeted sequence capture" data from 935 nuclear loci [17]. However, some phylogenetic uncertainties, as well as a few incongruences between nuclear and plastid inferences, still remain.

From the structural point of view, plastomes in angiosperms are 120-160 kb long and have a quadripartite structure with two single-copy regions (LSC, long, and SSC, short single copy) separated by two inverted repeats (IRA and IRB) [18]. Each plastid genome of Asteraceae investigated to date is around $150 \mathrm{~kb}$ long and contains ca. 80 protein-coding genes, four ribosomal RNAs (rRNA) and 30 transfer RNAs (tRNA) in the expected quadripartite organization [19]. Large changes in plastid DNA structure across land plants are unusual, but some families such as Geraniaceae, Fabaceae and Ericaceae, do show several plastome rearrangements (e.g., expansions, contractions, inversions or losses of an IR; [20]), in many cases reported as phylogenetically informative. In Asteraceae, all plastomes -excepting those from Barnadesioideae, a small basal subfamily with roughly 100 speciesshare a distinctive structural feature: a double inversion in the plastid DNA [14]. Both inversions are located in the LSC region, with the larger inversion ( $22.8 \mathrm{~kb}$ long) containing the second one $(\sim 3.3 \mathrm{~kb}$ long). These inversions have been confirmed using both Sanger and next-generation sequencing (NGS), the latter from a few species of the family (e.g., Artemisia capillaris, [21]). However, further studies including a larger representation of species could provide new insights into the structural variability of Asteraceae plastomes.

The advent of NGS technologies, as well as their continuously descending costs, have enabled the massive generation of genomic data for multiple species. In plants, whole genome sequencing (WGS) datasets frequently include plastid DNA data. The development of de novo assembly bioinformatic tools such as NovoPlasty [22] or SOAPdenovo2 [23], free and relatively easy to work with, have further facilitated the reconstruction of plastomes. Following those approaches, the plastid genomes of some species from Asteraceae have already been sequenced and published, now being stored in public repositories. However, this available genomic data represents a scattered and uneven taxonomic sampling, so more data is needed to analyse the diversity of plastomes within the family. In this study, we combine (i) seven previously published plastome reconstructions, (ii) 17 new plastome assemblies obtained from WGS raw data stored in repositories, and (iii) twelve new plastomes assembled from Illumina sequences specifically generated for this work. This strategy allowed us to build a comprehensive dataset with the whole plastid genome of 36 species representing the most important subfamilies and tribes of Asteraceae. We will use this data to thoroughly characterize the plastome variability of the family. We will also infer the most complete plastid phylogenomic reconstruction carried out in Asteraceae, comparing our results to previous phylogenetic and phylogenomic studies on the family. 


\section{Results}

\subsection{Plastome reconstruction in Asteraceae}

The mixed sampling strategy, combining Illumina sequences generated for this study and raw genomic data obtained from public repositories, resulted in 30 new plastomes for Asteraceae, completely reconstructed and circularized. All these plastomes have the standard structure typically found in angiosperms, comprising two copies of the IR region $(24,126$ to $25,245 \mathrm{bp})$, separated by the LSC $(82,297$ to $85,288 \mathrm{bp})$ and the SSC (17,859 to $18,786 \mathrm{bp}$ ) regions (Table 2). Plastid lengths were similar in all species analysed, being Achillea millefolium the species with the smallest plastid genome $(149,113 \mathrm{bp})$ and Melampodium linearilobum the biggest one $(153,872 \mathrm{bp})$. The GC content of the assembled plastomes ranged between $37 \%$ to $38.1 \%$. After all quality controls, the read coverage per nucleotide ranged between 10 to 6021 and the percentage of Ns in whole plastome reconstructions ranged between $0.00 \%$ to $3.80 \%$ (Table 1 ).

Table 1. Species information, SRA and GenBank accessions and mapping details for Asteraceae and the outgroup.

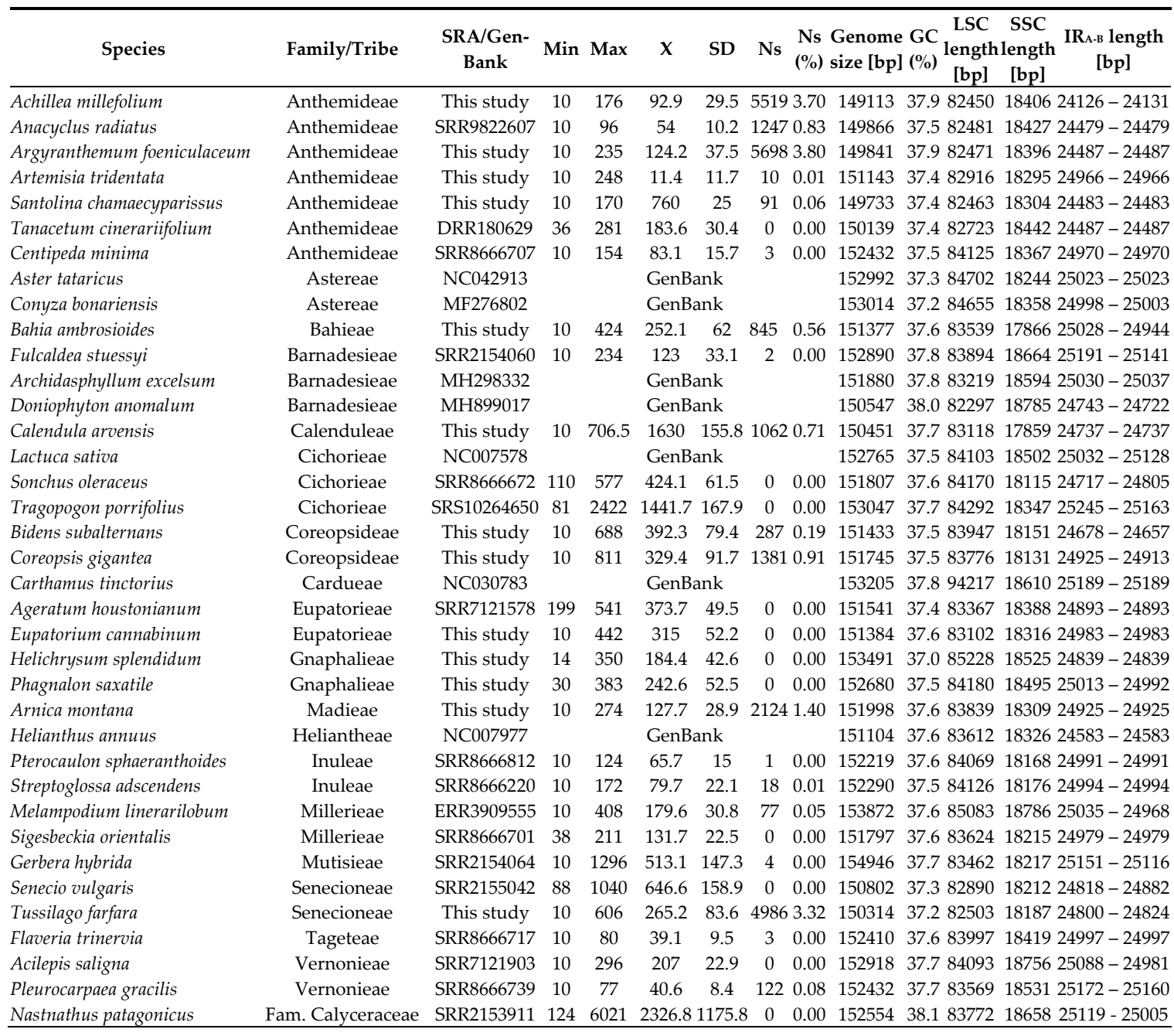

Asteraceae plastomes analysed in this study encode 80 CDS, 30 tRNAs and 4 rRNAs, with an overall of 114 genes (Table S1). Two canonical CDS were annotated as putative pseudogenes $(\Psi)$ based on their altered structure and significantly higher nucleotide diversity: $y c f 1\left(\mathrm{IR}_{\mathrm{B}}\right)$ and $r p l 19\left(\mathrm{IR}_{\mathrm{B}}\right)$. Plastomes analysed include 18 intron-containing genes,

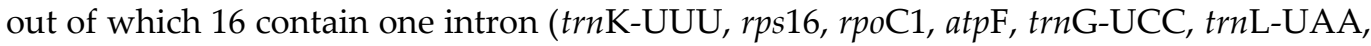

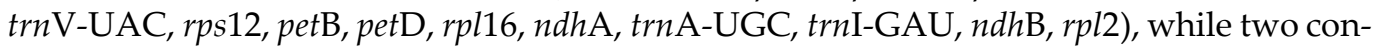
tain two introns (clpP and $y c f 3)$. 


\subsection{Phylogenetic analysis}

The resulting tree topology from Bayesian and ML inferences is shown in Figure 1. BI and ML trees were identical in topology (Figure S1), both phylogenetic approaches being highly resolved but BI showing stronger support in a few branches. In addition, phylogenetic inferences done with different combinations of CDS, tRNA and rRNA and species datasets (Supplementary Figure X) to validate the topology of the tree were congruent with the main topology result. The phylogenetic positions of all early divergent clades of the family Asteraceae (i.e., subfamilies Barnadesioideae, Mutisioideae and Carduoideae) are well-supported ( $p p=1, B S=100$, in all cases). The monophyly of subfamily Cichorioideae is also well-supported, being placed as the sister group of the subfamily Asteroideae $(\mathrm{pp}=1, \mathrm{BS}=100)$. The subfamily Asteroideae is divided into two major clades, one representing the Helianthodae supertribe and another constituted by supertribes Asterodae and Senecionodae [16, 42], here grouped in a fully supported clade. In the Helianthodae supertribe, the node grouping tribes Heliantheae and Millerieae only shows high support in $\mathrm{BI}$ tree ( $\mathrm{pp}=0.99, \mathrm{BS}=62)$, the same pattern occurring in the group constituted by tribes Eupatorieae and Madieae ( $p p=0.99, \mathrm{BS}=64$ ). In the Asterodae + Senecionodae clade, there are only two nodes that do not show the highest support according to both phylogenetic approaches: the split between tribe Gnaphalieae and tribes Anthemideae and Astereae $(\mathrm{pp}=0.91, \mathrm{BS}=60)$; and the branch grouping tribes Anthemideae and Astereae $(\mathrm{pp}=1, \mathrm{BS}$ $=67)$.

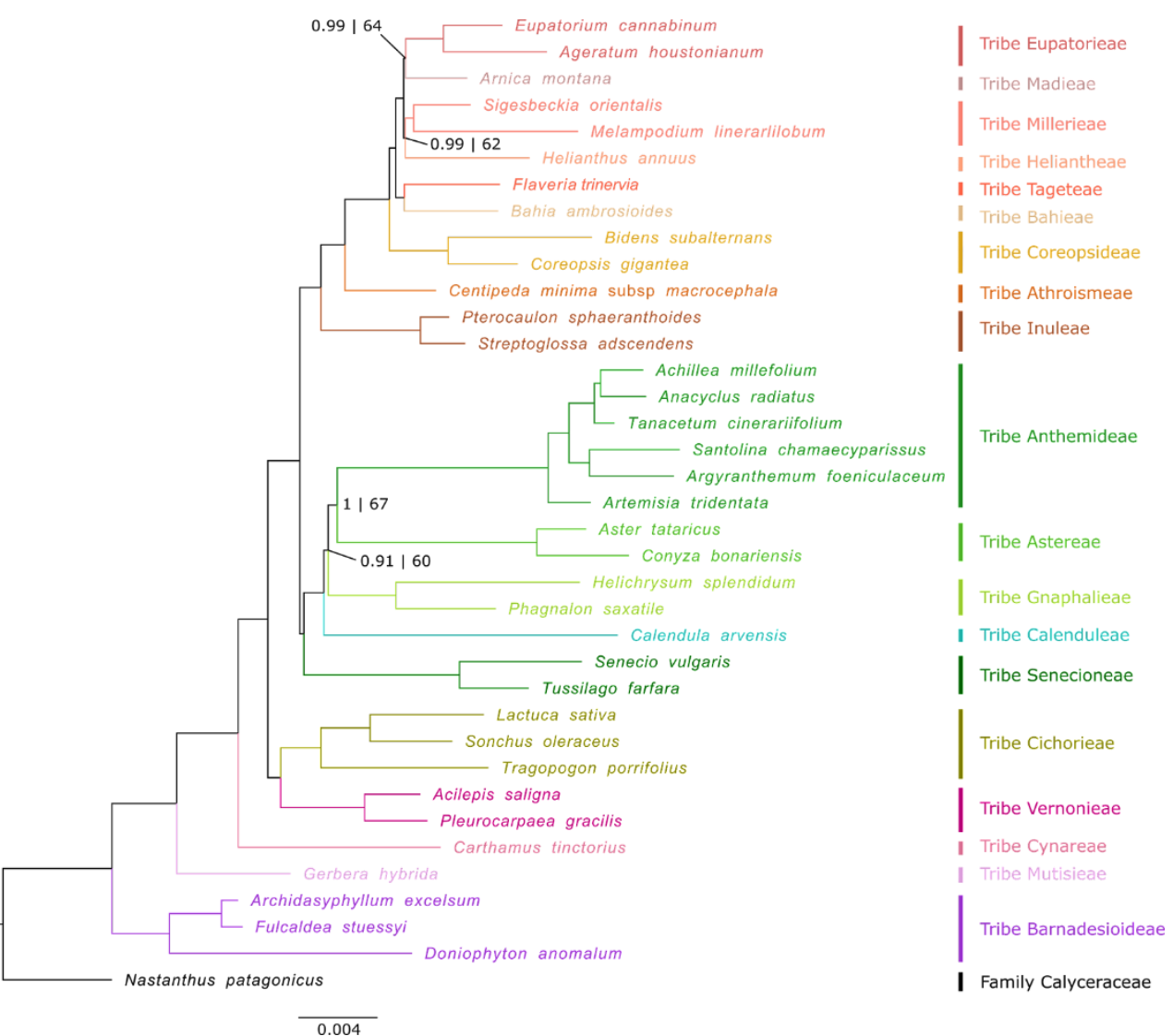

Figure 1. Phylogenomic tree of 37 species derived from 77,259 bp of the plastid coding DNA sequences (CDS), transfer RNAs (tRNA) and ribosomal RNAs (rRNA), using maximum likelihood (ML) and Bayesian inference (BI) approaches. Nodes without numbers are considered nodes with maximum support for both ML and BI approaches. Nodes with numbers are spots with low support in, at least, one phylogenetic inference approach (posterior probability/bootstrap). Low support is defined as: posterior probability $<1$; bootstrap $<70$. 


\subsection{Structural comparison of plastomes}

All Asteraceae plastomes, together with those of outgroups Nastanthus patagonicus (Calyceraceae), Scaevola taccada (Goodeniaceae) and Menyanthes trifoliata (Menyanthaceae), were used to perform a comparative analysis of plastid DNA structure across the MGCA clade and within family Asteraceae. The results obtained from this analysis (Figure 2, Figure S2) indicate that most Asteraceae plastomes exhibit a high level of sequence similarity and structural conservation. The analysis confirms the existence of a rearrangement in the LSC consisting of a double inversion: one large inversion of $\sim 22.8$ $\mathrm{kb}$, and one small inversion of $\sim 3.3 \mathrm{~kb}$ nested within the large one. This rearrangement is present in all species of subfamilies Mutisioideae, Carduoideae, Cichorioideae and Asteroideae, while it does not appear in subfamily Barnadesioideae nor in the sister family Calyceraceae (Figure 2). Family Menyanthaceae has the same plastome structure as Calyceraceae and the members of the tribe Barnadeiosideae. However, the plastome of the species representing Goodeniaceae, the sister family to the clade constituted by Calyceraceae and Asteraceae, is full of rearrangements - not only in the LSC region, but also in the SSC and in both IR - so its LSC structure could not be compared with the other families.
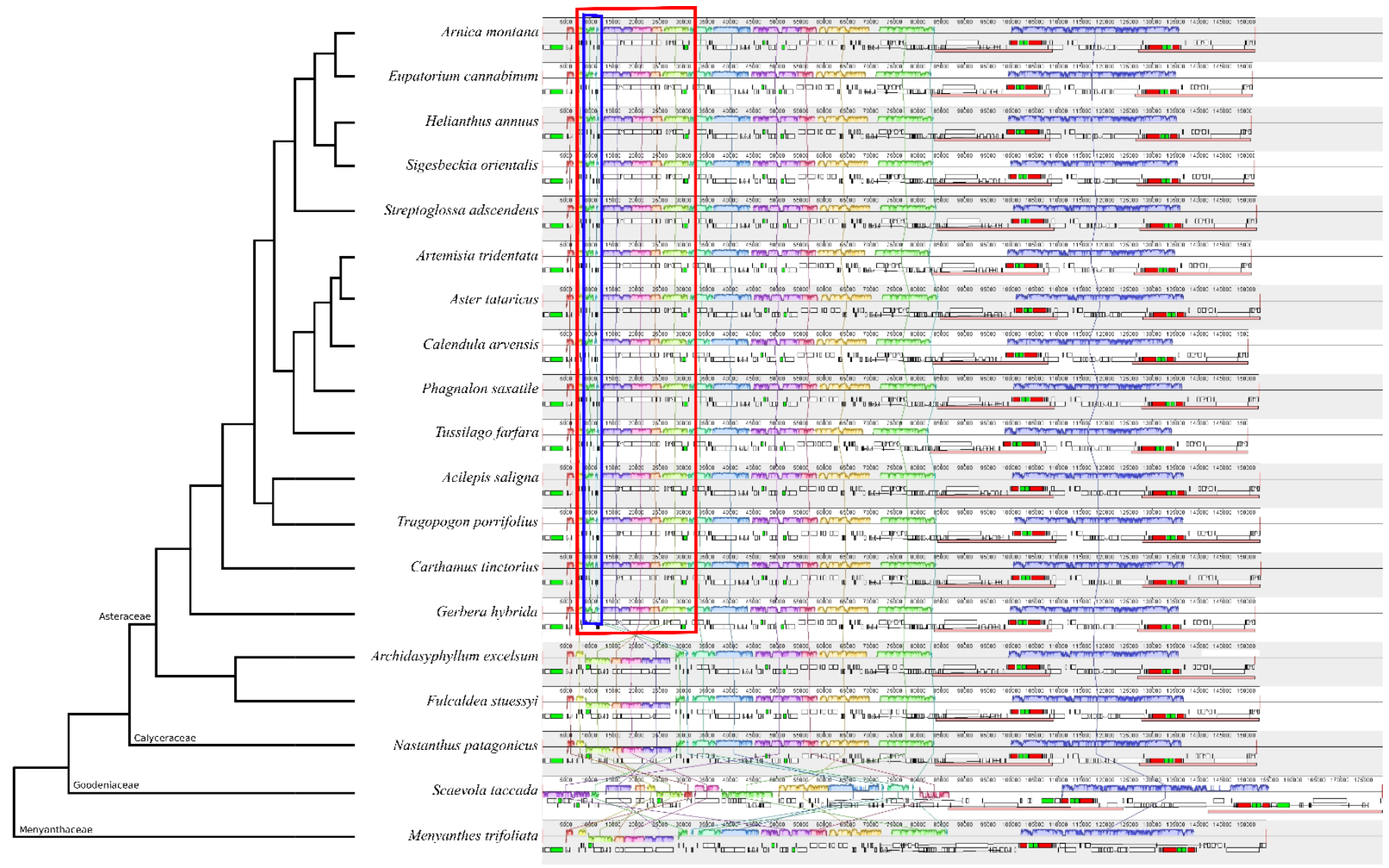

Figure 2. MAUVE alignment of a subset of 19 plastid genomes of species from family Asteraceae, Calyceraceae, Goodeniaceae and Menyanthaceae, sorted phylogenetically. Within each of the alignment, local collinear blocks are represented by blocks of the same color and connected by lines. Subfamily Barnadesioideae and family Calyceraceae have marked the region without the "double inversion" - In blue, the big inversion and, in red, the small inversion nested within the big one - 
By visualizing the expansions and contractions of the boundaries of the IR regions (Figure 3), we also found an additional structural difference in the plastome showed by the family Calyceraceae and the subfamily Barnadesioideae as compared to the plastomes of the remaining Asteraceae. Most Asteraceae species harbour the same genes in the boundaries between IR regions and single copy regions (LSC/IRA: rps19; IRA/SSC: ycf1; SSC/IRB: $\left.{ }^{\Psi} y c f 1 ; \mathrm{IR}_{\mathrm{B}} / \mathrm{LSC}:{ }^{\Psi} r p s 19\right)$. However, species from subfamily Barnadesioideae and the Calyceraceae Nastanthus patagonicus lack the ${ }^{\Psi}$ rps19 pseudogene localized in the boundary between the IRв and the LSC (Figure 3, Figure S3).

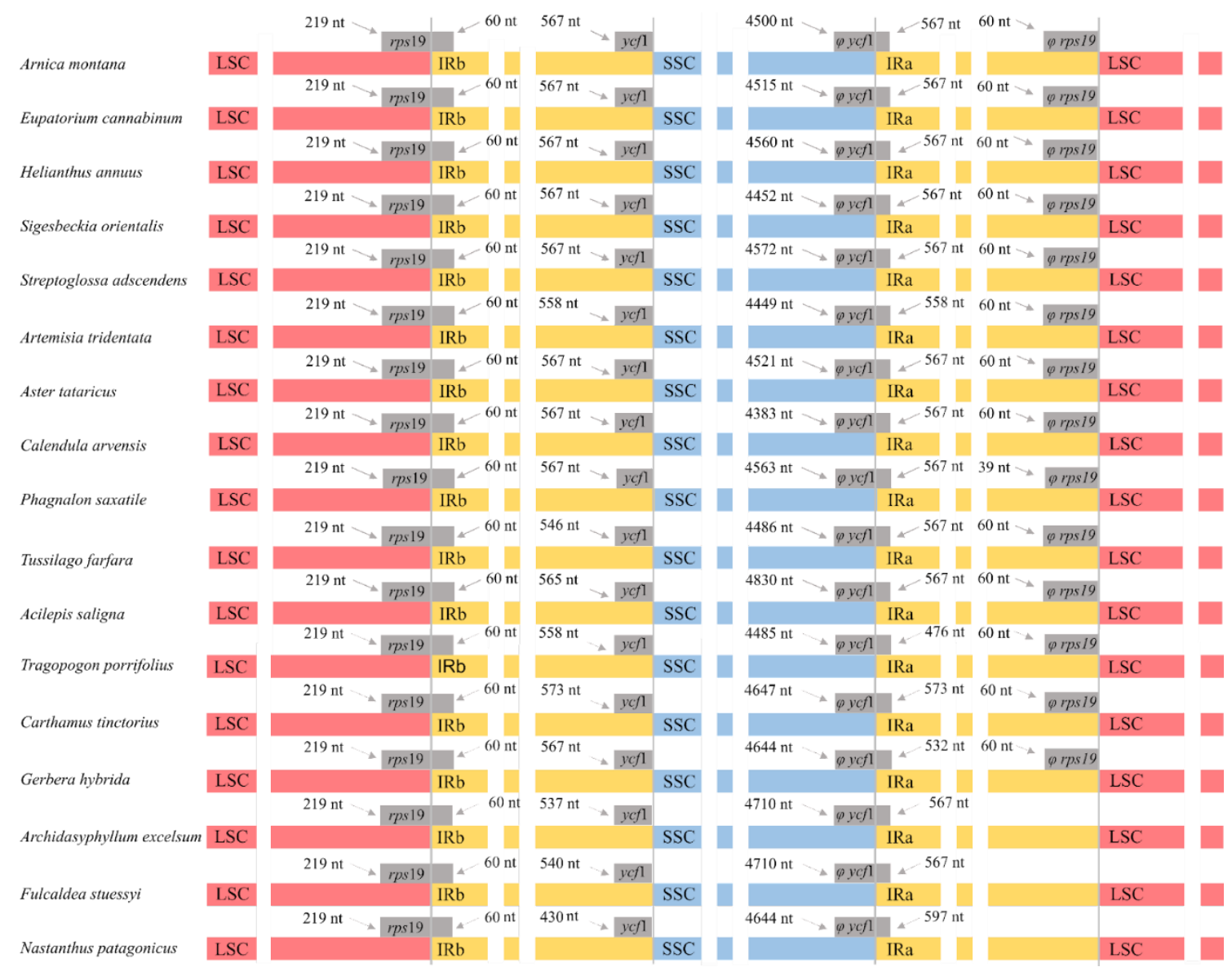

Figure 3. Comparison of the boundaries of the LSC, SSC and IR regions of a subset of 17 species from families Asteraceae and Calyceraceae (Nastanthus patagonicus). Genes suffixed with a phi $(\varphi)$ are potential pseudogenes.

\subsection{Characterization of sequence divergence, repeats and SSRs}

We compared coding genes, tRNA, rRNA and intergenic spacers across all Asteraceae species examined to find nucleotide divergence hotspots. For the 225 regions analysed (29 tRNA, 83 CDS, four rRNA and 109 intergenic spacers) the $\pi$ value within Asteraceae ranged from 0 ( $n d h \mathrm{~A}-n d h \mathrm{H}, t r n \mathrm{R}-\mathrm{ACG}, r p l 2-r p l 23)$ to 0.43814 (ycf1-ndhF) (Figure 4). Considering the whole family, most of the intergenic spacers could be regarded as highly divergent regions, surpassing the threshold of $\pi=0.05$, with the exception of the inverted repeat, where any region outweighs the threshold value. 


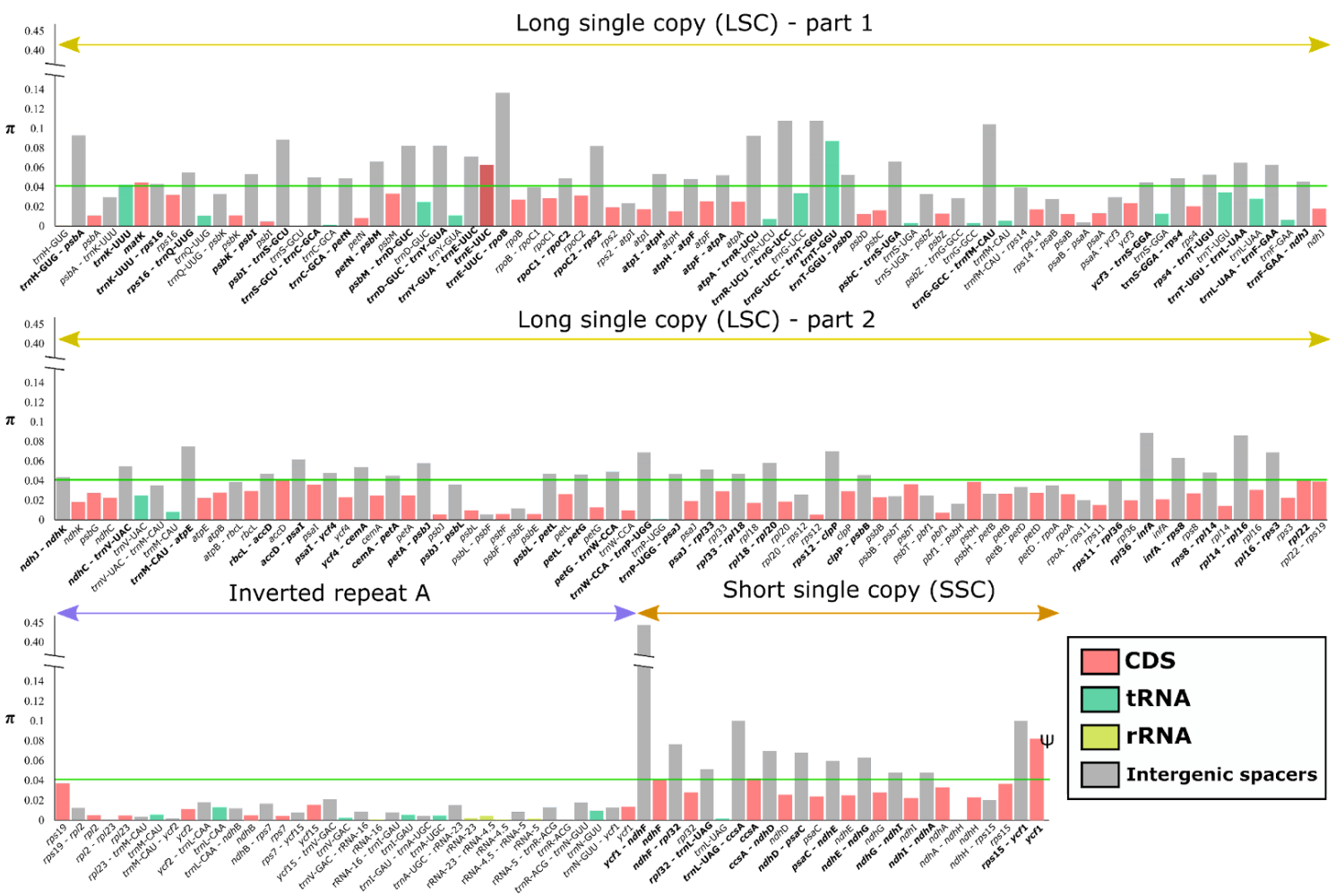

Figure 4. Nucleotide diversity $(\pi)$ through 36 species of family Asteraceae. Different coding and non-coding regions (CDS, tRNA, rRNA, IGS) are indicated by colours. The nucleotide diversity threshold (0.04) is indicated by the green line.

For the entire plastid genomes, the IR regions (IRA $\pi=0.00768$, IRB $\pi=0.00791$ ) were more conserved than both the LSC $(\pi=0.03611)$ and the SSC $(\pi=0.05974)$. Both IR recovered the same nucleotide diversity for almost all genes, being mirror images one of the other. Regarding to the different genomic regions analysed in this study, the tRNA and the rRNA were the regions with lower nucleotide diversity (tRNA $\pi=0.01558, \operatorname{rRNA} \pi=$ $0.00109)$, followed by the CDS $(\pi=0.02238)$ and the intergenic spacers $(\pi=0.04971)$.

We classified sequence dispersed repeat motifs into four categories: forward, reverse, palindromic, complement reverse and tandem repeats. In general, palindromic and reverse repeats were the most common, and a low proportion of complement reverse repeats was only identified in Aster tataricus (1), Bidens subalternans (2), Conyza bonariensis (1), Helianthus annuus (4), Helichrysum splendidum (2), Pleurocarpaea gracilis (3) and Archidasyphyllum excelsum (1) (Figure 54 ). Tandem repeats were distributed among all the species, from 18 (Arnica montana) to 54 (Conyza bonariensis) (Figure S5).

Microsatellites or SSRs were detected in every species analysed, and Asteraceae plastid genomes contained from 124 (Argyranthemum foeniculaceum) to 234 (Conyza bonariensis) microsatellites (Figure S5). All plastomes analysed had four types of SSRs (mono, di-, triand tetranucleotides), and a few also contained penta- and hexanucleotides (Supl. Figure $\mathrm{Yb}$ ). Most microsatellites were mononucleotides in all Asteraceae and Calyceraceae species (on average, mono-: $66.77 \%$, di-: $24.98 \%$, tri-: $2.81 \%$, tetra-: $4.40 \%$, penta-: $0.79 \%$, hexanucleotides: $0.25 \%)$. Almost all mononucleotides were highly AT-rich $(97.12 \%)$, and AT/TA repeats were also the most common among dinucleotide microsatellites (55.73\%). 


\section{Discussion}

In this study we have analysed 36 complete plastid genomes from species of a single family, 30 of which being de novo assembled for the first time. This extensive sampling has helped us understand the structural diversity of Asteraceae plastid genomes in a phylogenetic context, covering the most relevant tribes of the family. Our work also constitutes the most complete phylogenomic approach based on whole plastid genomes performed in Asteraceae, complementing the recent evolutionary studies of the family based on nuclear and plastid phylogenomic data.

\subsection{Structural and nucleotide diversity of Asteraceae plastomes}

The plastid genomes of the species here studied present the typical quadripartite structure and they are in the genome size range for land plants (Table 1, Figure 1) [43]. The length of plastomes included in our analyses showed a maximum variability of 4759 $\mathrm{bp}$, which is congruent with the length differences found in previous Asteraceae plastomes published in GenBank. Usually, plastid genomes exist in two different structural haplotypes within an individual, differing in the orientation of the LSC or of the SSC [44]. We have only detected one such structural haplotype in all the plastid genomes assembled in this study (Figure 2), most likely as an artifactual consequence of the plastome reconstruction method based on short reads; possibly, the use of long-reads would have revealed the existence of both haplotypes [45]. Previous comparative analysis of plastomes in Asteraceae show similar results regarding structure, genome size and haplotype found $[27,46,47]$. While the structure of all chloroplast genomes analysed was highly conserved, when early divergent Asteraceae (i.e., subfamily Barnadesioideae) were compared with the rest of the family, two re-arrangements were found in the LSC region (Figure 2): a large inversion of $\sim 22.8 \mathrm{~kb}$ and a smaller $\sim 3.3 \mathrm{~kb}$ inversion nested within it. This double inversion is localized in all major clades of Asteraceae, except in the early-diverging subfamily Barnadesioideae. The phylogenetic distribution of these re-arrangements is consistent with previous results based on restriction endonuclease digestions, PCR and Sanger sequencing [14, 48], which first detected these inversions and estimated that they originated during the late Eocene. In our study, we report for the first time that those Asteraceae species showing the double inversion in the LSC region (i.e. all of them excepting those belonging to Barnadesioideae) also present a pseudogenized $r p s 19$ at the end of the IRв. In contrast, Barnadesioideae subfamily as well as Calyceraceae family members show an alternative structure, lacking the pseudogenized rps19 (Figure 3) in one of the inverted repeats. Recent studies of plastome diversity show that the absence/presence of this pseudogene is scattered across angiosperms $[49,50,51]$. Our results suggest that this structural plastome feature (i.e. the presence of the pseudogenized rps19) might show a phylogenetic signal in Asteraceae, putatively co-occurring (or happening over a very short time span) with the double inversion event. However, this result has to be taken with caution, as [52] reported absence of the rps19 pseudogene in the Asteraceae species Artemisia annua, while showing its presence in all the other Asteraceae and Artemisia species there analysed.

The representative species from family Menyanthaceae (i.e., the sister family of Goodeniaceae, Calyceraceae and Asteraceae) seems to have a plastid genome structure similar to that found in Calyceraceae and in Barnadesioideae species. However, the representative of family Goodeniaceae included in the study shows a plastome structure completely altered by re-arrangements as compared to the rest of the families within the MGCA clade. As recently reported by [53], plastomes from Goodeniaceae have many intergenic regions with low GC proportion and many repeats, which may cause technical problems at the assembly level, possibly leading to an artefactual structure. Further work using new approaches of long read sequencing (e.g., Oxford Nanopore or PacBio 
technologies) could help to correctly assemble and explore the sequence of Goodeniaceae plastid genomes [54].

At the level of genetic diversity we found that intergenic spacers of Asteraceae have more than twice the nucleotide diversity of tRNA, rRNA and CDS, most likely because intergenic regions are not subject to selection constraints, allowing higher sequence divergence [55]. Regarding genic regions, tRNA and rRNA are more conserved than CDS, possibly due to the housekeeping function of the former two. There are also different levels of sequence conservativeness depending on the region, being IR sequences less variable than those of SSC and LSC, as reported previously [56, 57, 58]. The main reason could be, again, that IRs are harbouring important housekeeping genes such as structural ribosomal RNA genes ( $r r n 4.5, r r n 23$ and $r r n 16)$, highly conserved even in organisms with short IRs that possess only rRNA genes and few intergenic spacers, such as in some algae [59].

Due to the combination of fast and slow-evolving regions, uniparental inheritance and ease of amplification, plastid DNA markers are among the preferred targets of many phylogenetic and phylogeographic studies. Based on their relatively high nucleotide divergence, we have identified several genic regions that could be used as molecular markers in studies of Asteraceae species beyond the well-known CDS matK [60] and $n d h \mathrm{~F}$ [61], such as the CDS rpl22, accD, ccsA and $y c f 1$, and the tRNAs trnK-UUU, trnE-UUC, and trnT-GGU.

Repetitive sequences play an important role in plastome rearrangements, and could be useful to understand the evolution of plant species and sequence divergence [62]. The two main repeated motifs in plastomes are microsatellites and dispersed repeats [63]. The microsatellite data reported in our study can be useful as potential markers for evolutionary and genetic population analyses in Asteraceae $[64,65]$. Our results suggest that there are no relevant differences in the proportion of tandem repeats between tribes, and microsatellites are also in the expected range found in other studies, such as [63] (91 to 94 microsatellites in Chaenomeles), [57] (172 microsatellites in Hagenia) and [66] (116 microsatellites in Rubus).

\subsection{Asteraceae plyogenomics based on plastid DNA}

The availability of NGS data for an increasing number of species has hugely contributed to our understanding of the evolutionary relationships among organisms. Including data of 36 species from 19 tribes and 5 subfamilies of Asteraceae, this study represents the most comprehensive phylogenomic reconstruction using whole plastome data obtained to date in this family (114 loci, 70,000 nt). Previous phylogenetic works had also approached the evolution of this huge and complex family, among the most relevant: (1) a meta-tree analysis combining results at lower taxonomic levels from several research works, based in 10 plastid DNA and one nuclear DNA marker (ITS) [1] and representing all tribes and subfamilies; (2) a phylogenetic inference based on 12 plastid markers representing 13 subfamilies and 40 tribes (length 17,319 nt) [16], (3) an exhaustive phylogenomic study based on target sequence capture of 763 nuclear loci representing 13 subfamilies and 45 tribes (269,585 nt) [17] and (4) a phylotranscriptomic analysis with data from 243 species (13 subfamilies and 41 tribes) within the family [67].

Our phylogenomic reconstruction based on plastome data showed overall consistency with the backbone tree topologies obtained in those previous approaches. However, unlike in the inferences by $[1,16,17]$, we found a differential topology with strict support in basal nodes of subfamily Asteroideae, which could have important consequences for the taxonomy below the subfamily level. [1] placed tribe Senecioneae - the largest of the family, with ca. 150 genera and 3000 species - in a polytomy with the clade formed by the Inuleae + Athroismeae + Heliantheae Alliance (i.e., supertribe 
Helianthodae) and the clade containing Calenduleae + Gnaphalieae + Astereae + Anthemideae (i.e., Asterodae supertribe). [16] had previously found Senecioneae (there presented as supertribe Senecionodae) sister of Helianthodae and Asterodae supertribes, but the position lacked statistical support. [17] improved the resolution of important basal nodes in Asteroideae, merging Senecioneae with Anthemideae, Astereae, Gnaphalieae and Calenduleae (i.e., supertribe Asterodae). However, these authors found incongruences in the relationships of these five tribes among the trees they generated using different phylogenetic methods. While ML and BI yielded Calenduleae as an early diverging group of the remaining tribes, the pseudocoalescence analysis (ASTRAL) resulted in a high support for a different topology, with Senecioneae as the sister tribe to a clade of the four remaining Asterodae tribes. The same position of Senecioneae - despite not showing full statistical support - was obtained in the reconstruction of [67], also indicating that supertribe Senecionodae would not be monophyletic. Despite the limited sampling, our work provided full resolution and congruence among phylogenetic analyses for those basal nodes in subfamily Asteroideae, supporting the reconstructions obtained by Mandel et al. (2019) using the ASTRAL approach and by [67] using transcriptomic data; i.e. Senecioneae as sister tribe to the clade constituted by Anthemideae, Astereae, Gnaphalieae and Calenduleae (i.e., supertribe Asterodae). Therefore, results based on plastome data - congruently with the last phylogenomic reconstructions based on nuclear data - suggest that the supertribe system for the subfamily Asteroideae early proposed by [42, Robinson 2005] could be recovered, merging tribe Senecionae with the typical supertribe Asterodae.

Our phylogenetic inference also differs from previous systematic reconstructions of the family based on nuclear and plastid markers in the relationships among the tribes of the Heliantheae alliance. As already reported by other phylogenetic studies mainly based on plastid DNA markers, such as [1, 16], we found the important tribe Coreopsidae (550 spp.) in an early-diverging position within the Heliantheae alliance (Figure 1). In contrast, in the phylogenomic reconstructions based on nuclear data by [17] or [67], Coreopsidae is placed in a much derived position as sister tribe of Heliantheae s.s. There are other incongruences between plastid and nuclear genomes regarding the position of some tribes in the Heliantheae alliance. According to our results, as well as to other studies based on plastid DNA data (e.g. [16]), tribe Tageteae was sister to tribe Bahieae. In contrast, Tageteae was placed either as sister to Millerieae [17] or as sister to the weakly supported clade constituted by Madieae, Chaenactideae, Bahieae, Perityleae and Eupatorieae [67] in phylogenomic reconstructions based on nuclear DNA data. These incongruences between plastid and nuclear genome have been explained by potential hybridisation events involving plastid capture $[16,67]$. However, considering that the Heliantheae alliance is thought to be one of Asteraceae groups experiencing a faster radiation [17], incomplete lineage sorting could also be a possible source of phylogenetic incongruence [39].

\section{Materials and Methods}

\subsection{Taxon sampling}

To obtain a representation as even as possible along the family Asteraceae, a mixed strategy of data gathering from online genome repositories and de novo sequencing was followed (Table 1). First, the complete plastomes of Archidasphyllum excelsum [24], Aster tataricus [25], Carthamus tinctorius (GenBank: NC030783), Conyza bonariensis [26], Doniophyton anomalum (GenBank: MH899017), Helianthus annuus [27] and Lactuca sativa [28] were downloaded from GenBank. Besides, new assemblies were constructed using short-read archives (SRA) from WGS projects corresponding to 19 species, downloaded from the European Bioinformatics Institute (EMBL-EBI). Additionally, twelve plastomes were assembled from Illumina sequences newly generated for this study. As outgroup taxa, we assembled the plastome of Nastanthus patagonicus (Calyceraceae) and downloaded from GenBank the complete plastomes of Scaevola taccada (Goodeniaceae, 
GenBank: ) and Menyanthes trifoliata (Menyanthaceae, GenBank: ), these three families constituting -together with Asteraceae- the "MGCA (Menyanthaceae + Goodeniaceae + Calyceraceae + Asteraceae) clade" (APG IV, 2016).

\subsection{DNA preparation and sequencing}

The total DNA of 13 species (Table 1) was isolated from dried leaf material using either a modified CTAB protocol [29] or the E.Z.N.A® Plant DNA Kit (Omega Bio-tek, Inc., Norcross, Georgia, USA), depending on the quality and/or sufficient amount of material. Herbarium vouchers of the specimens are deposited at the Institut Botànic de Barcelona (IBB, CSIC-Ajuntament de Barcelona). The quality of each extraction was checked by spectrophotometry with Nanodrop 1000 (PeqLab, Erlangen, Germany) and the DNA concentration by fluorometry with Qubit Fluorometric Quantification (ThermoFisher Scientific, Waitham, Massachusetts, USA). De novo random sequencing of whole DNA was performed by Beijing Genomics Institute (BGI; Shenzhen, China), employing an Illumina HiSeq X10 (Illumina, San Diego, California, USA) platform, generating around 10 million paired-end reads (150 nt long) from 500 bp insert size fragment libraries (Supplementary Table 1).

\subsection{Genome assembly and annotation}

The quality of all raw sequencing data - 13 species sequenced for this study and 17 obtained from SRA - was assessed by FastQC version 0.11.9 ( http://www.bioinformatics.babraham.ac.uk/projects/fastqc/). Plastid genome reconstruction was performed with a mixed strategy combining de novo reconstruction of all plastomes and mapping assemblies of reads. De novo reconstruction of plastid sequences of 31 species was performed through the NOVOPlasty pipeline version 2.6.3 [22], using the raw whole dataset of Illumina reads, previously trimming the adapters, as recommended by the authors. After this de novo assembly, all raw data were additionally filtered based on the following rules: (i) adapter trimming; (ii) quality control: each read has $>90 \%$ of bases with a quality cut-off value of $>20$. These filtering steps were carried out using CLC Genomics Workbench 10.0.1 (CLC-BIO, Aarhus, Denmark). These high-quality reads were then mapped to the circular reconstructions obtained with NOVOPlasty using Geneious version 2020.1.1 (Biomatters, Auckland, New Zealand) with the mapping parameters by default, obtaining a consensus sequence for each species. All bases with $<10$ coverage were replaced by Ns.

Each plastome consensus was annotated using the software GeSeq [30] included in the platform MPI-MP CHLOROBOX (https://chlorobox.mpimp-golm.mpg.de/), selecting the options to perform tRNAscan-SE version 2.0.3 and BLAT, using the reference sequences that are more phylogenetically proximal available in NCBI Ref Seq (https://www.ncbi.nlm.nih.gov/refseq/). Subsequently, all automatically annotated consensus were checked manually using Geneious. The annotated plastid genomes have been submitted to GenBank.

\subsection{Plastome phylogenetic analyses}

To estimate the phylogenetic relationships in Asteraceae based on plastome data, 36 species of this family were analysed, together with one species of the sister family Calyceraceae (Nastanthus patagonicus) as outgroup, and discarding species from families Goodeniaceae and Menyanthaceae to avoid amiss alignments. Both Maximum Likelihood $(\mathrm{ML})$ and Bayesian Inference (BI) analyses were performed on a dataset of 37 plastome sequences including a single IR (i.e., removing one of the IR copies to avoid redundant information), the SSC and LSC regions. All genes present in the dataset were extracted separately, and then concatenated discerning between CDS, tRNA and rRNA; the noncoding regions were excluded from the analysis to be sure that all nucleotides are aligned 
with their homologous. The concatenated matrix was aligned using MAFFT version 7 [31], then manually checked with Geneious, resulting in a dataset of 77,259 nucleotide sites. The aligned dataset was partitioned by separating the coding genes from the tRNA and rRNA, as well as by categorizing the nucleotides in each CDS based on the position they occupy in a codon (first, second, or third). For the BI, the program MrBayes version 3.2.6 [32] included in the web-server CIPRES [33] (https://www.phylo.org/) was used to run two independent Markov chains Monte Carlo (MCMC) for 5,000,000 generations, with tree sampling every 1,000 generations. The average standard deviation was confirmed to be less than 0.01 and the potential scale reduction factor was near 1.0 for all parameters. For the ML inference, the program RAxML version 8.2.10 [34] was used with 1,000 bootstrap replicates and other parameters using the default settings. For both BI and ML approaches, PartitionFinder2 [35] was used to select the best evolutionary model for each concatenated region, chosen by selecting the scheme with the lowest AICc score. For the BI, the best partition scheme and substitution model was fitted for each region analysed (Table S2). Regarding ML inference, RAxML allows for only a single evolutionary model in partitioned analyses, which was selected according to PartitionFinder2 results (i.e. GTRGAMMA). For both inferences, the first $25 \%$ of the trees were discarded as 'burn-in' and the posterior probabilities/bootstraps were estimated constructing the $50 \%$ majority rule consensus tree.

To validate the topology obtained with the previous phylogenetic analysis and the support values obtained within the family Asteraceae, additional phylogenetic analysis using datasets with different combinations of genes and non-coding regions were performed. These subsets were: (i) CDS+tRNA+rRNA, (ii) CDS and (iii) the whole plastid genome sequences (Figure S1). Only the BI approach was used to perform the phylogenetic analysis for these datasets, employing the same parameter selection options mentioned above (Table S2).

\subsection{Plastome diversity analyses}

The complete plastomes of the Asteraceae analysed in this study were used to estimate the structural and nucleotide diversity of plastid DNA within the family. Plastome rearrangements were checked among Asteraceae and the phylogenetically close families Calyceraceae, Goodeniaceae and Menyanthaceae (i.e., the "MGCA clade"; APG IV, 2016), to explore if the double inversion in the plastid DNA -as well as other possible structural changes- are apomorphies only found in Asteraceae or if these rearrangements are also present in close families. Structural changes across plastid genomes of Asteraceae and proximal families were analysed via whole genome alignment in MAUVE version 2.4.0 [36], with the MAUVE algorithm using default parameters. The expansion and contraction of the inverted repeats (IR) boundaries were also explored in order to check if these regions show differential patterns in length and gene annotations across species.

Previously to all analyses performed, the dataset containing all plastomes was aligned using MAFFT version 7 aligner [31], and then manually adjusted using Geneious. For the screening of the genetic variability between species, the nucleotide diversity $(\pi)$ was estimated using DNAsp version 6 [37] for all coding DNA sequences (CDS), transfer RNA (tRNA), ribosomal RNA (rRNA) and intergenic spacers found in LSC, SSC and a single IR regions.

To characterize repeat sequences in the plastid genomes we used REPuter [38], with Hamming distance set at 3 and repeat range size from 30 to $90 \mathrm{bp}$, considering four types of repeats: forward, reverse and palindromic and complement sequences [39]. Tandem repeats were analysed using the Tandem Repeats Finder [40] with default parameters. Short sequence repeats (SSRs) were identified using the MISA microsatellite finder [41], with the following thresholds: eight repeat units for mono SSRs, four repeat units for 
di- and trinucleotide repeat SSRs, and three repeat units for tetra-, penta- and hexanucleotide repeat SSRs.

\section{Conclusions}

In conclusion, whole plastid genome data has been established as a powerful tool to understand evolutionary trends in Asteraceae, adding support to previous systematic inferences based on different markers. Our results confirmed the double inversion in cpDNA occurring during the early evolution of Asteraceae and revealed an additional structural change-appearance of a rps19 pseudogene- that could be evolutionarily linked to the two inversions. Our work has also contributed information about the gene composition, nucleotide diversity and repeat content in Asteraceae plastomes, which could be useful for the design of novel molecular markers for phylogeographic and population genetic studies. Finally, the phylogenomic reconstruction based on whole plastome data clarified previous uncertain questions on Asteraceae systematics at the tribe level, while also exposing some major incongruences among the evolutionary histories revealed by nuclear and plastid DNA data.

Supplementary Materials: The following are available online at www.mdpi.com/xxx/s1, Figure S1: Phylogeny reconstructed using Bayesian inference (BI) of family Asteraceae, representing the relationship among 18 tribes, excluding the subfamily Barnadesioideae and the outgroup Nastanthus patagonicus (family Calyceraceae). Three subsets had been used: (A) Coding DNA Sequences, (B) Coding DNA Sequences, transfer RNAs and ribosomal RNAs, (C) the whole plastid sequences, Figure S2. MAUVE alignment of 37 plastid genomes of species from family Asteraceae and Calyceraceae, sorted phylogenetically. Within each group, local collinear blocks are represented by blocks of the same color and connected by lines. The hypothetical origin of the rearrangement is represented by a star in the phylogenetic tree, Figure S3: Comparison of the boundaries of the LSC, SSC and IR regions of a subset of 37 species from family Asteraceae and Calyceraceae. Genes suffixed with a phi $(\varphi)$ are potential pseudogenes, Figure S4: Number of different long repeat sequence types found in the plastid genomes of the family Asteraceae and Calyceraceae, Figure S5. Number of SSR loci analysed and number of tandem repeats identified for each species of the family Asteraceae and Calyceraceae, Table S1: List of genes annotated in the studied plastid genomes of 37 species of Asteraceae and Calyceraceae, Table S2: Models used for phylogenetic analyses, Table S3: Microsatellites distribution, frequency and number for each species of this study.

Author Contributions: Conceptualization, J.P.P-D., S.G. and D.V.; methodology, J.P.P-D. and D.V.; software, J.P.P-D.; validation, J.P.P-D., S.G. and D.V.; formal analysis, J.P.P-D.; investigation, J.P.PD., S.G. and D.V.; resources, J.P.P-D., S.G. and D.V.; data curation, J.P.P-D., S.G. and D.V.; writing original draft preparation, J.P.P-D., S.G. and D.V.; writing - review and editing, J.P.P-D., S.G. and D.V.; visualization, J.P.P-D., S.G. and D.V.; supervision, J.P.P-D., S.G. and D.V.; project administration, S.G. and D.V.; funding acquisition, S.G. All authors have read and agreed to the published version of the manuscript.

Funding: This research was funded by the Dirección General de Investigación Científica y Técnica (Spanish Government: PID2020-119163GB-I00) and by the Generalitat de Catalunya ('Ajuts a grups de recerca consolidats' 2017SGR01116). SG benefited from a Ramón y Cajal contract (RYC-201416608) from the government of Spain.

Data Availability Statement: In this section, please provide details regarding where data supporting reported results can be found, including links to publicly archived datasets analyzed or generated during the study. Please refer to suggested Data Availability Statements in section "MDPI Research Data Policies" at https://www.mdpi.com/ethics. You might choose to exclude this statement if the study did not report any data.

Acknowledgments: We are grateful to Ales Kovarik, for providing sequence data for Tragopogon species.

Conflicts of Interest: The authors declare no conflict of interest.

\section{Supplementary Materials}




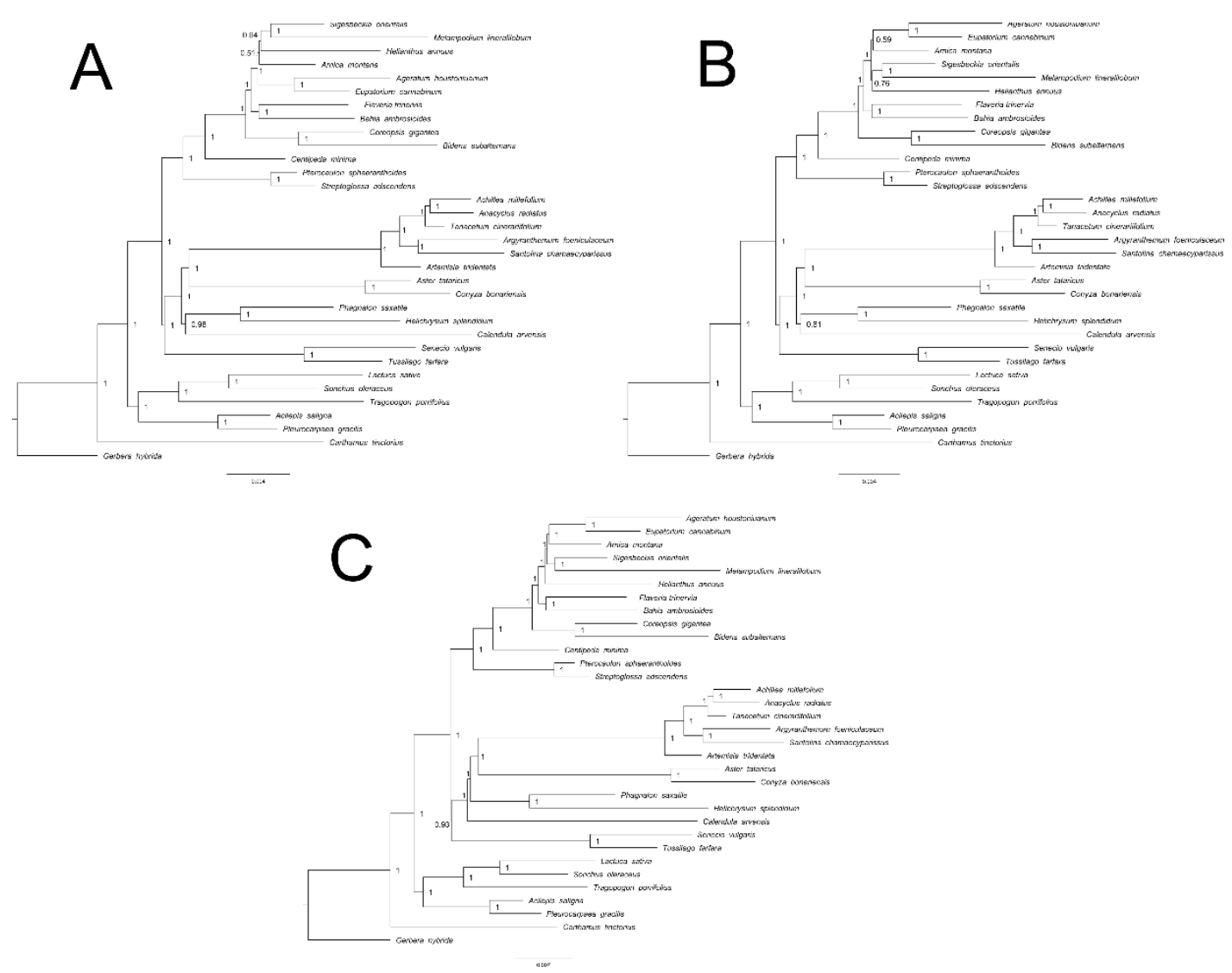

Figure S1. Phylogeny reconstructed using Bayesian inference (BI) of family Asteraceae, representing the relationship among 18 tribes, excluding the subfamily Barnadesioideae and the outgroup Nastanthus patagonicus (family Calyceraceae). Three subsets had been used: (A) Coding DNA Sequences, (B) Coding DNA Sequences, transfer RNAs and ribosomal RNAs, (C) the whole plastid sequences. 


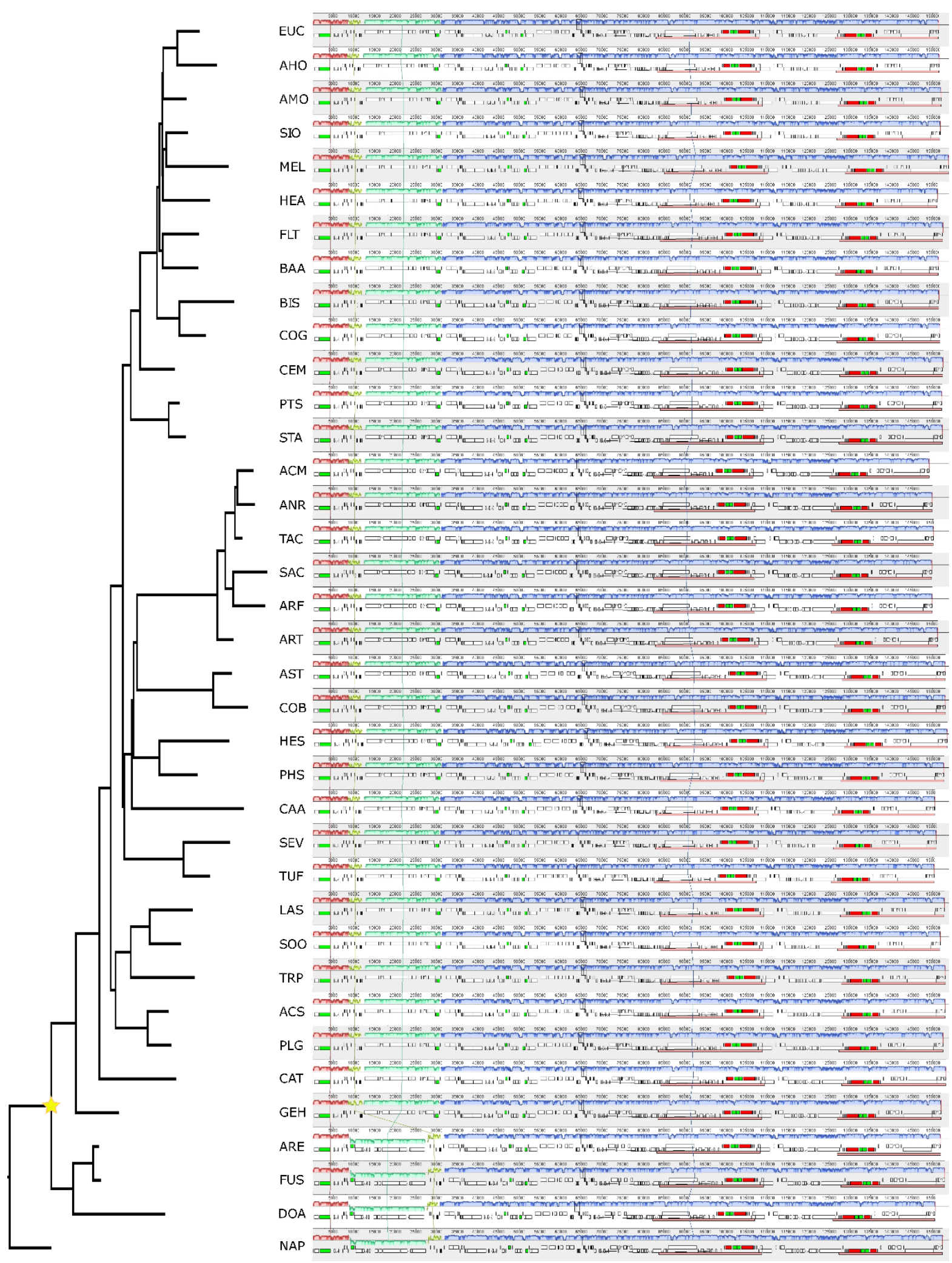

Figure S2. MAUVE alignment of 37 plastid genomes of species from family Asteraceae and Calyceraceae, sorted phylogenetically. Within each group, local collinear blocks are represented by blocks of the same color and connected by lines. The hypothetical origin of the rearrangement is represented by a star in the phylogenetic tree. 


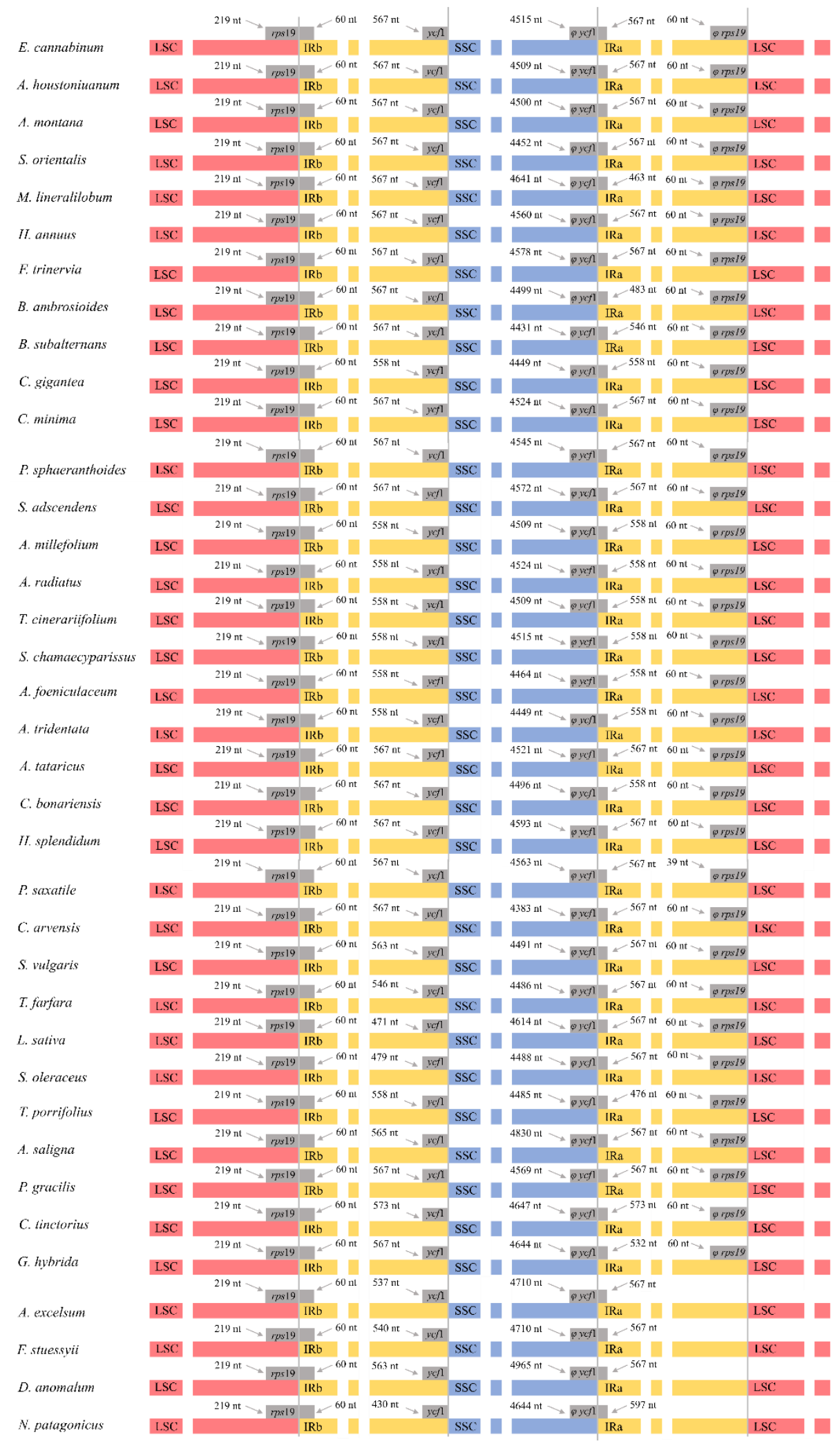

Figure S3. Comparison of the boundaries of the LSC, SSC and IR regions of a subset of 37 species from family Asteraceae and Calyceraceae. Genes suffixed with a phi $(\varphi)$ are potential pseudogenes. 


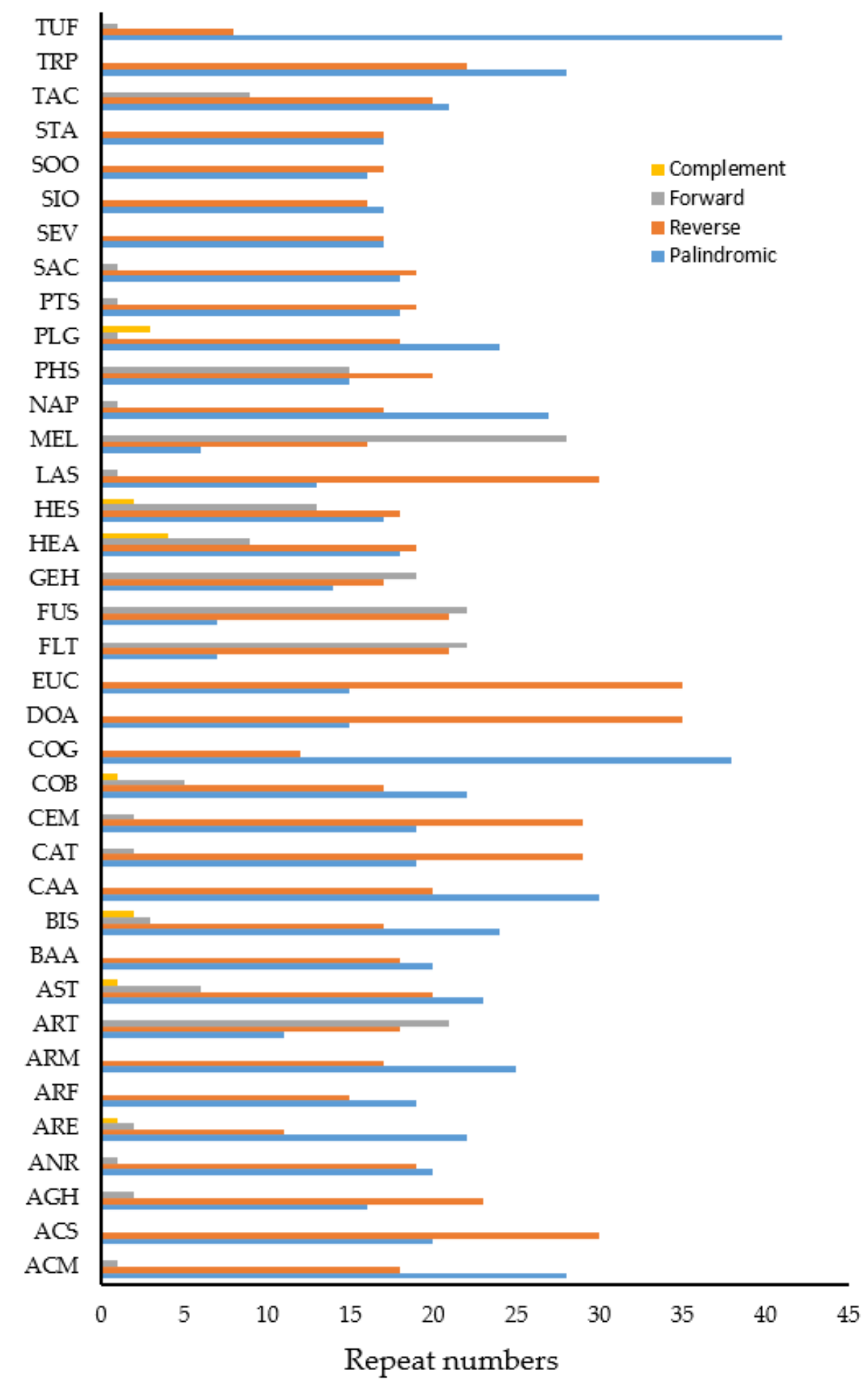

Figure S4. Number of different long repeat sequence types found in the plastid genomes of the family Asteraceae and Calyceraceae. 


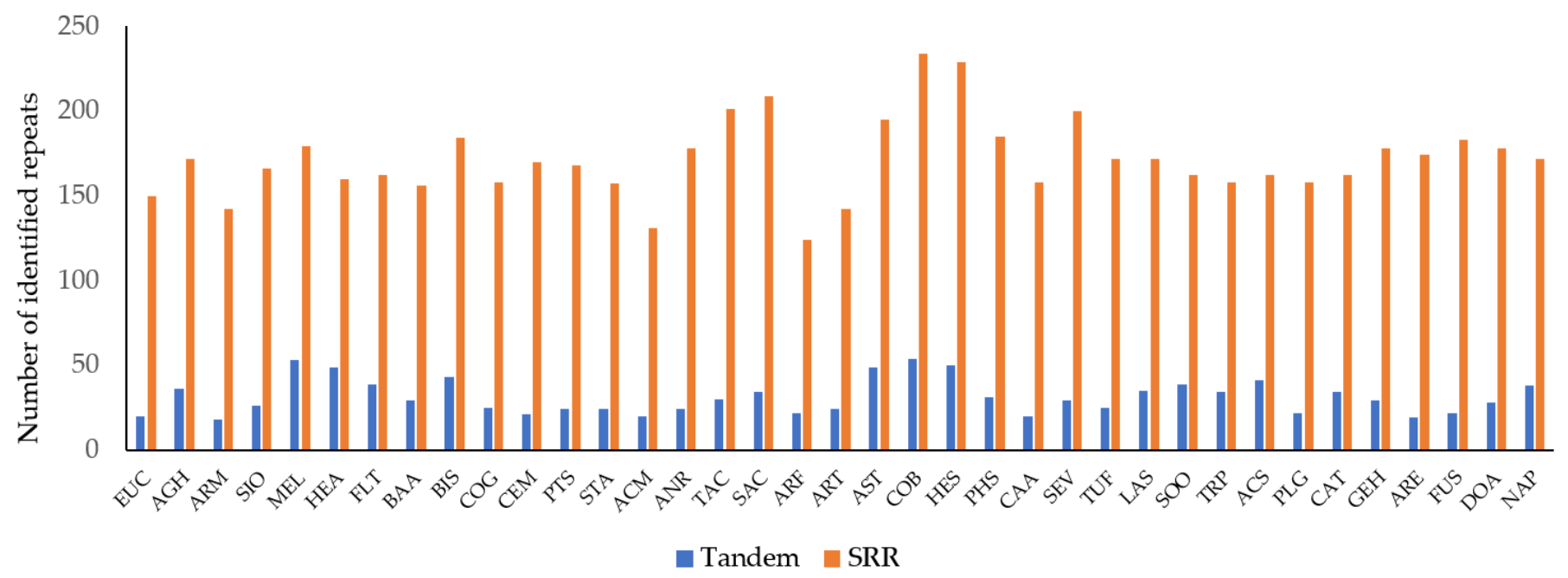

Figure S5. Number of SSR loci analysed and number of tandem repeats identified for each species of the family Asteraceae and Calyceraceae. 
Table S1. List of genes annotated in the studied plastid genomes of 37 species of Asteraceae and Calyceraceae

\begin{tabular}{|c|c|}
\hline Gene family & Gene \\
\hline Transfer RNA & 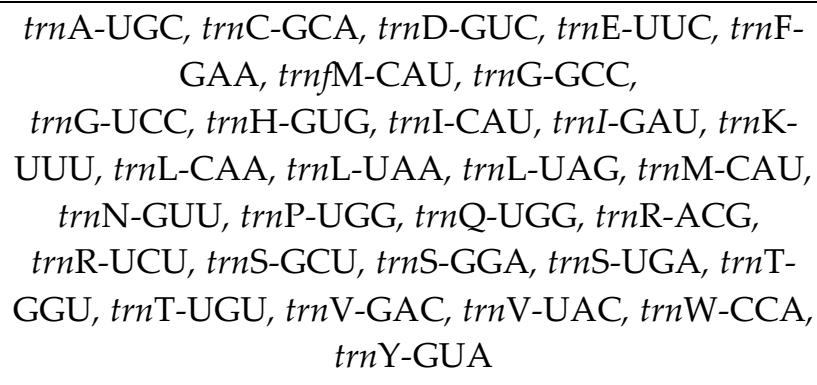 \\
\hline Small ribosomal units & $\begin{array}{c}r p s 2, r p s 3, r p s 4, r p s 7, r p s 8, r p s 11, r p s 12, r p s 14, r p s 15 \\
r p s 16, r p s 18, r p s 19\end{array}$ \\
\hline Large ribosomal units & rpl2, rpl14, rpl16, rpl20, rpl22, rpl23, rpl32, rpl33, rpl36 \\
\hline RNA polymerase sub-units & $r p o \mathrm{~A}, r p o \mathrm{~B}, r p o \mathrm{C} 1, r p o \mathrm{C} 2$ \\
\hline Translation initiation facto & infA \\
\hline NADH dehydrogenase & $\begin{array}{c}n d h \mathrm{~A}, n d h \mathrm{~B}, n d h \mathrm{C}, n d h \mathrm{D}, n d h \mathrm{E}, n d h \mathrm{~F}, n d h \mathrm{G}, n d h \mathrm{H}, \\
n d h \mathrm{I}, n d h \mathrm{~J}, n d h \mathrm{~K}\end{array}$ \\
\hline Photosystem I & $p b f 1, p s a \mathrm{~A}, p s a \mathrm{~B}, p s a \mathrm{C}, p s a \mathrm{I}, p s a \mathrm{~J}, y c f 3, y c f 4$ \\
\hline Photosystem II & $\begin{array}{c}p s b \mathrm{~A}, p s b \mathrm{~B}, p s b \mathrm{C}, p s b \mathrm{D}, p s b \mathrm{E}, p s b \mathrm{~F}, p s b \mathrm{H}, p s b \mathrm{I}, p s b \mathrm{~J} \\
p s b \mathrm{~K}, p s b \mathrm{~L}, p s b \mathrm{M}, p s b \mathrm{~T}, p s b \mathrm{Z}\end{array}$ \\
\hline Cytochrome b/f complex & $\operatorname{pet} \mathrm{A}, \operatorname{pet} \mathrm{B}, \operatorname{pet} \mathrm{D}, \operatorname{pet} \mathrm{G}, \operatorname{pet} \mathrm{L}, \operatorname{pet} \mathrm{N}$ \\
\hline ATP synthase & $\operatorname{atp} \mathrm{A}, \operatorname{atp} \mathrm{B}, \operatorname{atp} \mathrm{E}, \operatorname{atp} \mathrm{F}, \operatorname{atp} \mathrm{H}, \operatorname{atp} \mathrm{I}, y c f 2$ \\
\hline Large subunit of rubisco & $r b c \mathrm{~L}$ \\
\hline Maturase & matK \\
\hline Protease & $c l p \mathrm{P}$ \\
\hline Acetyl-CoA-carboxylase sub-unit & $\operatorname{acc} \mathrm{D}$ \\
\hline Envelope membrane protein & $\operatorname{cem} \mathrm{A}$ \\
\hline Component of TIC complex & Ycf1 \\
\hline c-Type cytochrome synthesis & $\operatorname{ccs} \mathrm{A}$ \\
\hline Uncharacterized protein & $y c f 15$ \\
\hline Ribosomal RNA & $r r n 4.5, r r n 5, r r n 16, r r n 23$ \\
\hline
\end{tabular}

Table S2. Models used for phylogenetic analyses.

\begin{tabular}{|c|c|c|c|c|c|}
\hline \multirow{2}{*}{ Inference Method } & \multicolumn{3}{|c|}{ Coding regions (CDS) } & \multirow{2}{*}{ tRNA } & \multirow{2}{*}{ rRNA } \\
\hline & Codon 1 & Codon 2 & Codon 3 & & \\
\hline Maximum Likelihood & $\mathrm{GTR}+\gamma$ & $\mathrm{GTR}+\gamma$ & $\mathrm{GTR}+\gamma$ & $\mathrm{GTR}+\gamma$ & $\mathrm{GTR}+\gamma$ \\
\hline Bayesian Inference & GTR + inv_r $\gamma$ & GTR + inv_r $\gamma$ & GTR + inv_r $\gamma$ & GTR + inv_r $\gamma$ & $\mathrm{K} 80+$ inv_r $\gamma$ \\
\hline
\end{tabular}

Table S3. Microsatellites distribution, frequency and number for each species of this study. 


\section{References}

1. Funk, V.A.; Anderberg, A.A.; Baldwin, B.G.; Bayer, R.J.; Bonifacino, J.M.; Breitwieser, I.; Brouillet, L.; Carbajal, R.; Chan, R.; Coutinho, A.X.P.; Crawford, D.J.; Crisci, J.V.; Dillon, M.O.; Freire, S.E.; Galbany-Casals, M.; Garcia-Jacas, N.; Gemeinholzer, B.; Gruenstaeudl, M.; Hansen, H.V.; Himmelreich, S.; Kaderei, J.W.; Källersjö, M.; Karaman-Castro, V.; Karis, P.O.; Katinas, L.; Keeley, S.C.; Kilian, N.; Kimball, R.T.; Lowrey, T.K.; Lundberg, J.; McKenzie, R.J.; Tadesse, M.N.; Mort, M.E.; Nordenstam, B.; Oberprieler, C.; Santiago, Ortiz, Pelser, P.B.; Randle, C.P.; Robinson, H.; Roque, N.; Sancho, G.; Semple, J.C.; Serrano, M.; Stuessy, T.F.; Susanna, A.; Matthew, Unwin, Urbatsch, L.; Urtubey, E.; Vallès, J.; Vogt, R.; Wagstaff, S.; Ward, J.; Watson, L.E. Compositae metatrees: the next generation. In Systematics, Evolution and Biogeography of Compositae; V.A. Funk, A. Susanna, T.F. Stuessy, R.J. Bayer, Eds.; International Association for Plant Taxonomy 2009; pp. 747-777.

2. Kadereit J.W.; Jeffrey, C. Flowering plants. Eudicots Asterales. In The Families and Genera of Vascular Plants; Kubitzki, K., Ed.; Springer-Verlag: Berlin/Heidelberg, Germany 2007, pp. 635.

3. Stuessy, T.F.; Spooner, D.M.; Evans, K.A. Adaptive significance of ray corollas in Helianthus grosseserratus (Compositae). Am Midl Nat 1986, 115(1), 191-197.

4. Anderberg, A.A.; Baldwin, B.G.; Bayer, R.G.; Breitwieser, J.; Jeffrey, C.; Dillon, M.O.; Eldenäs, P.; Funk, V.; Garcia-Jacas, N.; Hind, D.J.N.; et al. Compositae. In Flowering Plants, Eudicots: Asterales. The Families and Genera of Vascular Plants; Kubitzki, K., Kadereit, J.W., Jeffrey, C., Eds.; Springer: Berlin/Heidelberg, Germany 2007; Volume VIII.

5. Semple, J.C.; Watanabe, K. A review of chromosome numbers in Asteraceae with hypotheses on chromosomal base number evolution. In Systematics, Evolution and Biogeography of Compositae; V.A. Funk, A. Susanna, T.F. Stuessy, R.J. Bayer, Eds.; International Association for Plant Taxonomy 2009; pp. 61-72.

6. Barker, M.S.; Li, Z.; Kiddler, T.I.; Reardon, C.R.; Lai, Z.; Oliveira, L.O.; Scacitelli, M.; Rieseberg, L.H. Most Compositae (Asteraceae) are descendants of a paleohexaploid and all share a paleotetraploid ancestor with the Calyceraceae. Am J Bot 2016, 103(7), 1203-1211.

7. Barber, J.C.; Finch, C.C.; Francisco-Ortega, J.; Santos-Guerra, A.; Jansen, R.K. Hybridization in Macaronesian Sideritis (Lamiaceae): evidence from incongruence of multiple independent nuclear and chloroplast sequence datasets. Taxon. 2007 56(1), 7488.

8. Jones, K.E.; Reyes-Betancort, J.A.; Hiscock, S.J.; Carine, M.A. Allopatric diversification, multiple habitat shifts, and hybridization in the evolution of Pericallis (Asteraceae), A Macaronesian endemic genus. Am J Bot 2014, 101(4), 637-651.

9. Garcia, S.; Panero, J.L.; Siroky, J.; Kovarik, A. Repeated reunions and splits feature the highly dynamic evolution of $5 S$ and $35 S$ ribosomal RNA genes (rDNA) in the Asteraceae family. BMC Plant Biol 2010, 10(1), 1-18.

10. Panero J.L.; Francisco-Ortega, J.; Jansen, R.K.; Santos-Guerra, A. Molecular evidence for multiple origins of woodiness and a New World biogeographic connection of the Macaronesian Island endemic Pericalis (Asteraceae: Senecioneae). P Natl Acad Sci USA 1999, 96(24), 13886-13891.

11. Jabaily, R.S.; Shepherd, K.A.; Gardner, A.G.; Gustafsson, M.H.; Howarth, D.G.; Motley, T.J. Historical biogeography of the predominantly Australian plant family Goodeniaceae. J Biogeogr 2014, 41(11), 2057-2067.

12. Denham, S.S.; Zavala-Gallo, L.; Johnson, L.A.; Pozner, R.E. Insights into the phylogeny and evolutionary history of Calyceraceae. Taxon 2016, 65(6), 1328-1344.

13. Kim, K.J.; Jansen, R.K.; Wallace, R.S.; Michaels, H.J.; Palmer, J.D. Phylogenetic implications of $r b c L$ sequence variation in the Asteraceae. Ann Mo Bot Gard 1992, 428-445.

14. Kim, K.J.; Choi, K.S.; Jansen, R.K. Two chloroplast DNA inversion originated simultaneously during the early evolution of the sunflower family (Asteraceae). Mol Biol Evol 2005, 22(9), 1783-1792.

15. Shaw, J; Lickey, E.B.; Schilling, E.E.; Small, R.L. Comparison of whole chloroplast genome sequences to choose noncoding regions for phylogenetic studies in angiosperms: the tortoise and the hare III. Am J Bot 2007, 94(3), 275-288.

16. Panero, J.L.; Crozier, B.S. Macroevolutionary dynamics in the early diversification of Asteraceae. Mol Phylogenet Evol 2016, 99, 116-132.

17. Mandel, J.R.; Dikow, R.B.; Siniscalchi, C.M.; Thapa, R.; Watson, L.E.; Funk, V.A. A fully resolved backbone phylogeny reveals numerous dispersals and explosive diversifications throughout the history of Asteraceae. P Natl Acad Sci USA 2019, 116(28), 14038-14088.

18. Mower, J.P.; Vickrey, T.L. Structural diversity among plastid genomes of land plants. In Advances in Botanical Research; Chaw, S.M., Jansen, R.K., Eds.; Elsevier, Ltd.: Amsterdam, Netherlands 2018, pp. 263-292

19. Loeuille B.; Thode, V.; Siniscalchi, C.; Andrade, S.; Rossi, M.; Pirani, J.R. Extremely low nucleotide diversity among thirty-six new chloroplast genome sequences from Aldama (Heliantheae, Asteraceae) and comparative chloroplast genomics analyses with closely related genera. PeerJ 2021, 9, e10886.

20. Ruhlman, T.A.; Jansen, R.K. Aberration or analogy? The atypical plastomes of Geraniaceae. In Advances in Botanical Research; Chaw, S.M., Jansen, R.K., Eds.; Elsevier, Ltd.: Amsterdam, Netherlands 2018, pp. 223-262.

21. Lee, Y.S.; Park, J.Y.; Kim, J.K.; Lee, H.O.; Park, H.S.; Lee, S.C.; Kang, J.H.; Lee, T.J.; Hung, S.H.; Yang, T.J. The complete chloroplast genome sequences of Artemisia gmelinii and Artemisia capillaris (Asteraceae). Mitochondrial DNA Part B 2021, 1(1), 410-411.

22. Dierckxsens, N.; Mardulyn, P.; Smits, G. NOVOPlasty: de novo assembly of organelle genomes from whole genome data. Nucleic Acids Res 2017, 45(4), e18-e18. 
23. Luo, R.; Liu, B.; Xie, Y.; Huang, W.; Yuan, J.; He, G.; Chen, Y.; Pan, Q.; Liu, Y.; Tang, J.; Wu, G.; Zhang, H.; Shi, Y.; Liu, Y.; Yu, C.; Wang, B.; Lu, Y.; Han, C.; Cheung, D.W.; Yiu, S-M.; Peng, S.; Xiaoqian, Z.; Liu, G.; Liao, X.; Li, H. Y.; Wang, J.; Lam, T-W.; Wang, J. SOAPdenovo2: an empirically improved memory-efficient short-read de novo assembler. Gigascience 2012, 1(1), 2047$217 X$.

24. Gruenstaeudl, M.; Jenke, N. PACVr: Plastome assembly coverage visualitzation in R. BMC Bioinformatics 2020, $21,1-21$.

25. Shen, X.; Guo, S.; Yin, Y.; Zhang, J.; Yin, X.; Liang, C.; Wang, Z.; Huang, B.; Liu, Y.; Xiao, S.; Zhu, G. Complete chloroplast genome sequence and phylogenetic analysis of Aster tataricus. Molecules 2018, 23(10), 2426.

26. Hereward, J.P.; Werth, J.A.; Thornby, D.F.; Keenan, M.; Chauhan, B.S.; Walter, G.H. Complete chloroplast genome of glyphosate resistant Conyza bonariensis (L.) Cronquist from Australia. Mitochondrial DNA Part B 2017, 2(2), 444-445.

27. Timme, R.E.; Kuehl, J.V.; Boore, J.L. A comparative analysis of the Lactuca and Helianthus (Asteraceae) plastid genomes: identification of divergent regions and categorization of shared repeats. Am J Bot 2007, 94(3), 302-312.

28. Kanamoto, H.; Yamashita, A.; Okumura, S.; Hattori, M.; Tomizawa, K.I. The complete genome sequence of the Lactuca sativa (lettuce) chloroplast. Plant Cell Physiol 2004, 45, S39-S39.

29. Dolye, I.J.; Doyle J.L. A rapid DNA isolation procedure for small quantities of fresh leaf tissue. No. Research 1987.

30. Tillich, M.; Lehwark, P.; Pellizzer, T.; Ulbricht-Jones, E.S.; Fischer, A.; Bock, R.; Greiner, S. GeSeq-versatile and accurate annotation of organelle genomes. Nucleic Acids Res 2017, 45(W1), W6-W11.

31. Katoh, K.; Standley, D.M. MAFFT multiple sequence alignment software version 7: improvements in performance and usability. Mol Biol Evol 2013, 30(4), 772-780.

32. Ronquist, F.; Teslenko, M.; Van Der Mark, P.; Ayres, D.L.; Darling, A.; Höhna, S.; Larget, B.; Liu, L.; Suchard, M.A.; Huelsenbeck, J.P. MrBayes 3.2: efficient bayesian phylogenetic inference and model choice across a large model space. Syst Biol 2012, 61(3), 539-542.

33. Miller, M.A.; Pfeiffer, W.; Schwartz, T. Creating the CIPRES Science Gateway for Inference of Large Phylogenetic Trees. Gatew. Comput. Environ. Work GCE 2010, 1-8.

34. Stamatakis, A. RAxML-VI-HPC: maximum likelihood-based phylogenetic analyses with thousands of taxa and mixed models. Bioinformatics 2006, 22(21), 2688-2690.

35. Lanfear, R.; Frandsen, P.B.; Wright, A.M.; Senfeld, T.; Calcott, B. PartitionFinder 2: new methods for selecting partitioned models of evolution for molecular and morphological phylogenetic analyses. Mol Biol Evol 2017, 34(3), 772-773.

36. Darling, A.C.; Mau, B.; Blattner, F.R.; Perna, N.T. MAUVE: multiple alignment of conserved genomic sequence with rearrangements. Genome Research 2004, 14(7), 1394-1403.

37. Rozas, J.; Ferrer-Mata, A.; Sánchez-DelBarrio, J.C.; Guirao-Rico, S.; Librado, P.; Ramos-Onsins, S.E.; Sánchez-Gracia, A. DNASP 6: DNA sequence polymorphism analysis of large datasets. Mol Biol Evol 2017, 34(12), 3299-3302.

38. Kurtz, S.; Choudhuri, J.V.; Ohlebusch, E.; Schleiermacher, C.; Stoye, J.; Giegerich, R. REPuter: the manifold applications of repeat analysis on a genomic scale. Nucleic Acids Res 2001, 29(22), 4633-4642.

39. Sun, M.; Soltis, D.E.; Soltis, P.S.; Zhu, X.; Burleigh, J.G.; Chen, Z. Deep phylogenetic incongruence in the angiosperms clade Rosidae. Mol Phylogenet Evol 2015, 83, 156-166.

40. Benson, G. Tandem Repeat Finder: a program to analyze DNA sequences. Nucleic Acids Res 1999, $27(2), 573-580$.

41. Beier, S.; Thiel, T.; Münch, T.; Scholz, U.; Mascher, M. MISA-web: a web server for microsatellite prediction. Bioinformatics 2017, 33(16), 2583-2585.

42. Robinson, H. New supertribes Helianthodae and Senecionodae, for the subfamily Asteroideae (Asteraceae). Phytologia 2004, 86(3), 116-120.

43. Xiao-Ming, Z.; Junrui, W.; Li, F.; Sha, L.; Hongbo, P.; Lan, Q.; Jing, L.; Yan, S.; Weihua, Q.; Lifang, Z.; Yunlian, C.; Qingwen. Inferring the evolutionary mechanism of the chloroplast genome size by comparing whole-chloroplast genome sequences in seed plants. Sci Rep-UK 2017, 7(1), 1-10.

44. Palmer J.D.; Stein, D.B. Conservation of chloroplast genome structure among vascular plants. Curr Genet 1986, $10(11), 823-833$.

45. Wang, W.; Lanfear, R. Long-reads reveal that the chloroplast genome exists in two distinct versions in most plants. Genome Biol Evol 2019, 11(12), 3372-3381.

46. Walker, J.F.; Zanis, M.J.; Emery, N.C. Comparative analysis of complete chloroplast genome sequence and inversion variation in Lasthenia burkei (Madieae, Asteraceae). Am J Bot 2014, 101(4), 722-729.

47. Wang, M.; Cui, L.; Feng, K.; Deng, P.; Du, X.; Wan, F.; Weining, S.; Nie, X. Comparative analysis of Asteraceae chloroplast genomes: structural organization, RNA editing and evolution. Plant Mol Biol Rep 2015, 33(5), 1526-1538.

48. Jansen, R.K.; Palmer, J.D. A chloroplast DNA inversion marks an ancient evolutionary split in the sunflower family (Asteraceae). P Natl Acad Sci USA 1987, 84(16), 5818-5822.

49. Wang, R.J.; Cheng, C.L.; Chang, C.C.; Wu, C.L.; Su. T.M.; Chaw, S.M. Dynamics and evolution of the inverted repeat-large single copy junctions in the chloroplast genome of monocots. BMC Evol Biol 2008, 8(1), 1-14.

50. Ni, L.; Zhao, Z.; Xu, H.; Chen, S.; Dorje, G. Chloroplast genome structures in Gentiana (Gentianaceae), based on three medicinal alpine plants used in Tibetan herbal medicine. Curr Genet 2017, 63(2), 241-252.

51. Ma, Q.; Li, S.; Bi, C.; Hao, Z.; Sun, C.; Ye. N. Complete chloroplast genome sequence of a major economic species, Ziziphus jujuba (Rhamnaceae). Curr Genet 2017, 63(1), 117-129.

52. Shen, X.; Wu, M.; Liao, B.; Liu, Z.; Bai, R.; Xiao, S.; Li, X.; Zhang, B.; Xu, J.; Chen, S. Complete chloroplast genome sequence and phylogenetic analysis of the medicinal plant Artemisia annua. Molecules 2017, 22(8), 1330. 
53. Nevill, P.G.; Zhong, X.; Tonti-Filippini, J.; Byrne, M.; Hislop. M.; Thiele, K.; van Leeuwen, S.; Boykin, L.M.; Small, I. Large scale genome skimming from herbarium material for accurate plant identification and phylogenomics. BMC Plant Methods 2020 16, 1.

54. Belser, C.; Istace, B.; Denis, E.; Dubarry, M.; Baurens, F.C.; Falentin, C.; Genete, M.; Berrabah, W.; Chèvre, A.M.; Delourme, R.; Deniot, G.; Denoeud, F.; Duffé, P.; Engelen, S.; Lemainque, A.; Manzanares-Dauleux, M.; Martin, G.; Morice, J.; Noel, B.; Vekemans, X.; D’Hont, A.; Rousseau-Gueutin, M.; Barbe, V.; Cruaud, C.; Wincker, P.; Aury, J.M. Chromosome-scale assemblies of plant genomes using nanopore long reads and optical maps. Nature Plants 2018, 4(11), 879-887.

55. Small, R.L.; Ryburn, J.A.; Cronn, R.C.; Seelanan, T.; Wendel, J.F. The tortoise and the hare: choosing between noncoding plastome and nuclear Adh sequences for phylogeny reconstruction in a recently diverged plant group. Am J Bot 1998, 85(9), 13011315.

56. Asaf, S.; Khan, A.L.; Khan, A.R.; Waqas, M.; Kang, S.M.; Khan, M.A.; Lee, S.M.; Lee, I.J. Complete chloroplast genome of Nicotiana otophora and its comparison with related species. Frontier in Plant Science 2016, 7, 843.

57. Gichira, A.W.; Avoga, S.; Li, Z.; Hu, G.; Wang, Q.; Chen, J. Comparative genomics of 11 complete chloroplast genomes of Senecioneae (Asteraceae) species: DNA barcodes and phylogenetics. Bot Stud 2019, 60(1), 17.

58. Wang, H.; Liu, X.; Moore, M.J.; Landrein, S.; Liu, B.; Zhu, Z.X.; Wang, H.F. Plastic phylogenomic insights into the evolution of the Caprifoliaceae sl (Dipsacales). Mol Phylogenet Evol 2020, 142, 106641.

59. Fang, J.; Lin, A.; Yuan, X.; Chen, Y.; He, W.; Huang, J.; Zhang, X.; Lin, G.; Zhang, J.; Xue, T. The complete chloroplast gen ome of Isochrysis galbana and comparison with related haptophyte species. Algal Research, 2020, 50, 101989.

60. Koch, M.; Haubold, B.; Mitchell-Olds, T. Molecular systematics of the Brassicaceae: evidence from coding plastidic matK and nuclear Chs sequences. Am J Bot, 2001, 534-554.

61. Bogler, D.J.; Pires, J.C.; Francisco-Ortega, J. Phylogeny of Agavaceae based on $n d h \mathrm{~F}$, rbcL and ITS sequences. Aliso: A Journal of Systematic and Evolutionary Botany, 2006, 22(1), 313-328.

62. Milligan, B.G.; Hampton, J.N.; Palmer, J.D. Dispersed repeats and structural reorganization in subclover chloroplast DNA. Mol Biol Evol, 1989, 6(4), 355-368.

63. Sun, J.; Wang, Y.; Liu, Y.; Xu, C.; Yuan, Q.; Guo, L.; Huang, L. Evolutionary and phylogenetic aspects of the chloroplast genome of Chaenomeles species. Sci Rep-UK, 2020, 10(1), 1-10.

64. Xu, D.; Abe, J.; Gai, J.; Shimamoto, Y. Diversity of chloroplast DNA SSRs in wild and cultivated soybeans: evidence for multiple origins of cultivated soybean. Theor Appl Genet, 2002, 105(5), 645-653.

65. Mariotti, R.; Cultrera, N.G.; Díez, C.M.; Baldoni, L.; Rubini, A. Identification of new polymorphic regions and differentiation of cultivated olives (Olea europaea L.) through plastome sequence comparison. BMC Plant Biol, 2010, 10(1), 1-13.

66. Yang, J.Y.; Pak, J.H.; Kim, S.C. The complete plastome sequence of Rubus takesimensis endemic to Ulleung Island, Korea: Insights into molecular evolution of anagenetically derived species in Rubus (Rosaceae). Gene, 2018, 668, 221-228.

67. Zhang, C.; Huang, C.H.; Liu, M.; Hu, Y.; Panero, J.L.; Luebert, F.; Gao, T.; Ma, H. Phylotranscriptomic insights into Asteraceae diversity, polyploidy, and morphological innovation. J Integr Plant Biol, 2021, 63(7), 1273-1293. 